\title{
Not So Innocent: Thrombocytopenia After One Dose of Rifampicin
} Çok da Masum Değil: Tek Doz Rifampisin Sonrası Derin Trombositopeni

\author{
Tarana NAGIYEVA' ${ }^{1}$, Meltem Işıkgöz TAŞBAKAN², Hüsnü PULLUKÇU², Oğuz Reşat SiPAHi², Tansu YAMAZHAN² \\ ${ }^{1}$ Ege University Faculty of Medicine, Department of Internal Medicine, Izmir, Turkey \\ ${ }^{2}$ Ege University Faculty of Medicine, Department of Infectious Diseases and Clinical Microbiology, Izmir, Turkey
}

Keywords: Rifampicin, thrombocytopenia, adverse effect, cat bite, rifampin

Anahtar Kelimeler: Rifampisin, trombositopeni, yan etki, kedi ısırığı, rifampin

\section{Dear Editor,}

Drug side effects are commonly encountered by physicians. Drugs may have different side effects which can manifest with varying clinical presentations ranging from generally mild allergic reactions to life-threatening conditions. Side effects may occur after the first dose or with repeated doses. Drug-induced thrombocytopenia is a rare side effect caused by the interaction of drug-dependent antibodies with platelet membrane glycoproteins. Numerous drugs may cause this condition, but the most common are carbamazepine, ibuprofen, quinine, oxaliplatin, rifampicin, sulfamethoxazole, trimethoprim, and vancomycin $^{[1]}$.

Rifampicin is a bactericidal antibiotic that is often used in the treatment of diseases such as tuberculosis and brucellosis. The most common side effects are dizziness, headache, numbness, abdominal pain, vomiting, pruritus, and skin rash. Elevated liver enzymes and toxic hepatitis are other common conditions ${ }^{[2]}$. Rifampicin-induced thrombocytopenia was first reported in $1970^{[3]}$. It is typically seen in high-dose intermittent treatment and is believed to be due to an immunological mechanism ${ }^{[3]}$. In this paper, we present a patient who developed urticaria and thrombocytopenia following a single dose of rifampicin.

A 59-year-old female patient presented to the emergency department with complaints of fever and swelling, redness, and limitation of motion in the distal phalanx of the second finger of her left hand five days after being bitten by a cat. She was diagnosed with soft tissue infection and treatment with amoxicillin/clavulanate $(875 / 125 \mathrm{mg})$ was initiated. The patient's symptoms had not improved after ten days of treatment, so fusidic acid (500 mg three times daily) was added to the antibiotic treatment. Laboratory tests done during this time were normal except for C-reactive protein level of $0.7 \mathrm{mg} / \mathrm{dL}(0.57-1.11)$ and sedimentation rate of $33 \mathrm{~mm} /$ hour. Her platelet count at that visit was $245,000 / \mathrm{L}$. The patient also did not respond to this treatment, and presented to the outpatient clinic five days later. Her treatment was changed to fusidic acid (500 mg three times daily) and oral rifampicin (600 mg/day). The patient was also evaluated by the Orthopedics Department for possible abscess/ osteomyelitis and radiographs were performed. Suspecting soft tissue infection, we decided to continue medical treatment under close follow-up. Laboratory tests during this period indicated no pathology except leukocytosis and high sedimentation rate. Forty-five minutes after initiating treatment with rifampicin, the patient started itching; two hours later, extensive urticaria appeared in addition to the itching and the patient admitted to the Emergency Service. After evaluation, the patient was transferred to Infectious Diseases Clinic with the diagnosis of skin/soft tissue infection and drug side effects. Physical examination revealed no pathology other than erythematous, blanching maculopapular skin lesions covering her entire body (Figure 1) and swelling, redness, pain, and limitation of motion in the affected finger. Severe thrombocytopenia $\left(18 \times 10^{3} / \mathrm{L}\right)$ was detected in laboratory tests. On peripheral blood smear, platelet

Cite this article as: Nagiyeva T, Taşbakan MI, Pullukçu H, Sipahi OR, Yamazhan T. Not So Innocent: Thrombocytopenia After One Dose of Rifampicin. Mediterr J Infect Microb Antimicrob. 2017;6:11.

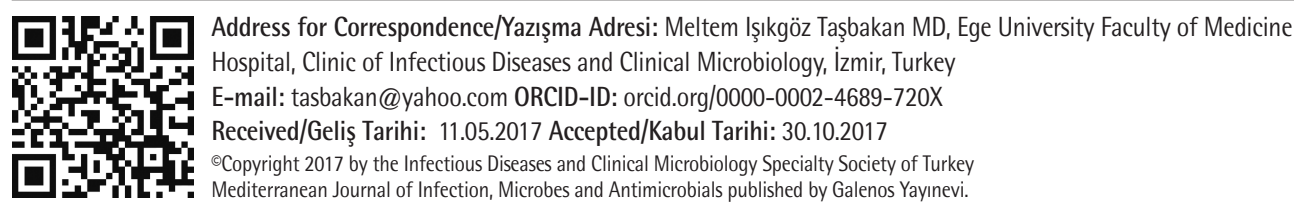

Mediterranean Journal of Infection, Microbes and Antimicrobials published by Galenos Yayinevi.
Presented in: This study was presented as a poster at the $6^{\text {th }}$ annual congress of the Infectious Diseases and Clinical Microbiology Specialty Society of Turkey (EKMUD), held on 11-15 May, 2016.

Published: 21 November 2017 
count was $40 \times 10^{3} / \mathrm{L}$ and large thrombocytes were evident. Due to the absence of any signs of acute hemorrhage, platelet replacement therapy was not administered. The former antibiotic treatment was discontinued and ampicillin/sulbactam (8 g/day) treatment was initiated. Immunologic (ANA, direct, indirect Coombs, C3, C4), serologic (hepatitis B, C, Rose Bengal, EpsteinBarr virus, cytomegalovirus, HIV, parvovirus, Rubella), and parasitological (toxoplasmosis, Leishmaniasis) tests for possible causes of secondary thrombocytopenia were all negative. Iron and total iron binding capacity, vitamin B12, and folic acid levels were normal. There was no pathology in the abdominal ultrasound except hepatosteatosis. The patient, who had never taken rifampicin before, had platelet counts of $32 \times 10^{3} / \mu \mathrm{L}$ in whole blood and $40 \times 10^{3} / \mu \mathrm{L}$ in the peripheral smear 48 hours discontinuing rifampicin therapy. Additional therapy was not considered due to regression of the thrombocytopenia. Hand radiographs taken to check for bone infection showed no signs of osteomyelitis. The patient's platelet increased to $263 \times 10^{3} / \mu \mathrm{L}$ by day ten, at which time the ampicillin/sulbactam treatment was completed and she was discharged.

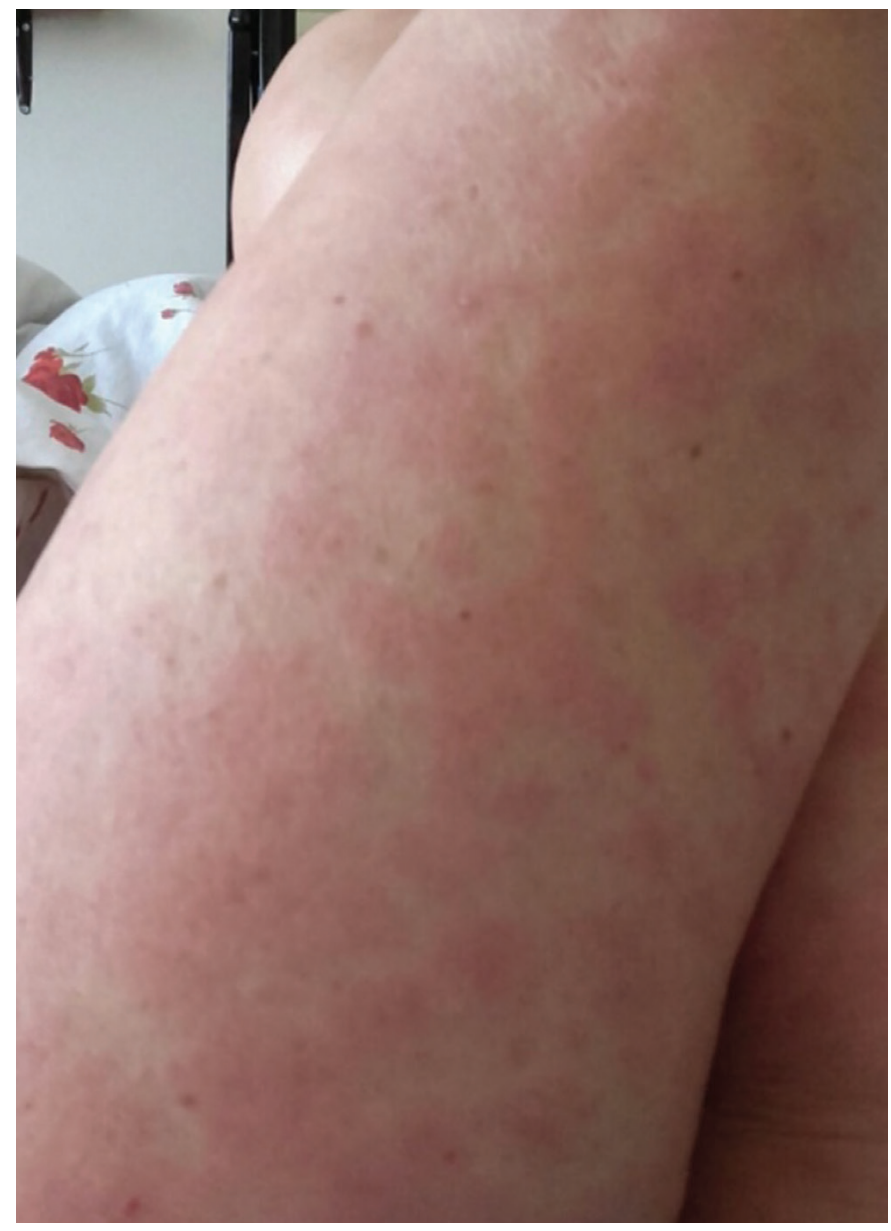

Figure 1. Erythematous, blanching maculopapular skin lesions covering the patient's entire body observed during physical examination
Various side effects can be encountered during antibiotic therapy. It is necessary to know the potential side effects that may arise during the use of antibiotics and to properly follow patients using them. Rifampicin, which is active against numerous bacteria, acts by inhibiting DNA-dependent RNA polymerase. It is usually included in combination therapies because it can rapidly lead to resistance when used alone ${ }^{[2]}$. Approximately $5-10 \%$ of patients treated with rifampicin experience drug side effects. Side effects are more common when used at high doses and intermittently ${ }^{[3]}$. They are also more common when using rifampicin in combination with clarithromycin, ciprofloxacin, and isoniazid. The combined use of rifampicin and isoniazid, the main drugs of tuberculosis treatment regimens, has been reported to increase the frequency of side effects, especially in children $^{[2]}$.

Drug-induced thrombocytopenia is generally associated with suppressed platelet production in the bone marrow or immune mechanisms. Several diagnostic criteria have been determined for drug-induced thrombocytopenia:

i) The patient developed thrombocytopenia after using the drug,

ii) Platelet levels return to normal after drug is discontinued,

iii) Prior to onset of thrombocytopenia, the drug was used alone or, if used in combination, thrombocytopenia regressed when that drug was discontinued,

iv) Other causes of thrombocytopenia have been excluded ${ }^{[1]}$.

Although thrombocytopenia can occasionally be seen in rifampicin treatment, it usually occurs as a result of longterm therapy and appears 1-2 weeks after starting the drug. Diagnosis is established based on thrombocytopenia onset with initiation of rifampicin therapy and resolution after discontinuing rifampicin (loss of antibodies after three weeks of cessation), and the exclusion of other causes. Our patient was also diagnosed after other causes of thrombocytopenia had been excluded and the thrombocytopenia regressed when rifampicin was discontinued.

Side effects usually appear after 1-2 weeks of drug use. However, the fact that the patient had no history of rifampicin use, developed redness, urticaria, and pruritus shortly after taking the drug, and recovered rapidly after stopping it all suggested that rifampicin was the cause. Developing immune thrombocytopenia after the first dose of rifampicin is rare phenomenon. Nevertheless, immune thrombocytopenia has been reported at up to $6 \%$ in two-day high-dose antituberculous therapy due to rifampicin-dependent antibodies ${ }^{[4]}$. IgG and $\lg \mathrm{M}$ antibodies against rifampicin play a role in the development of immune thrombocytopenia ${ }^{[3]}$. These antibodies adhere to platelets and disrupt their structure. Some studies have shown that antibodies produced as a result of daily rifampicin use 
remain in the body without inducing an allergic reaction. In patients receiving intermittent treatment, antibodies produced during off periods cause an allergic reaction when treatment is resumed ${ }^{[5]}$. Some studies have shown that the interaction between drug-dependent antibodies and the platelet membrane protein glycoprotein Ib-IX leads to immunological thrombocytopenia ${ }^{[6,7]}$. It is possible to detect drug-dependent antibodies with advanced laboratory tests. The identified antibodies show $49-66 \%$ sensitivity, 78-92\% specificity, and mean positive predictive values of about $80-83 \%$. However, a negative test result is not sufficient to exclude the diagnosis ${ }^{[8]}$. The drug-dependent antibody levels of our patient could not be assessed because this analysis is not available in our hospital. This is a limitation of this case report. Cases of thrombocytopenia due to anti-tuberculous drugs used in tuberculosis therapy have also been reported in the literature ${ }^{[9]}$. These cases are believed to be a result of immune mechanisms and the thrombocytopenia usually resolves within 7-10 days without treatment. Although no marked symptoms appear with slight reductions in platelet count, spontaneous hemorrhages requiring intervention can occur when the thrombocyte count drops below $12 \times 10^{3} / \mu$, and hemorrhage can be life-threatening at platelet counts below $6 \times 10^{3} / \mu{ }^{[3]}$. Steroid therapy, plasmapheresis, and platelet transfusion are needed for patients who have platelet counts of 10,000-20,000 and are at risk for hemorrhage. In patients who develop purpura after the first dose, rifampicin should be discontinued immediately and should not be given again, even at low doses. Our patient did not require platelet replacement because her platelet count started to recover after rifampicin treatment was discontinued.

In summary, caution should be exercised when prescribing any drug because side effects may manifest with different clinical presentations. Although the most common side effects may be known, it is also important to monitor patients and perform the necessary analyses to recognize the less frequently encountered side effects of a drug. In addition, this case was reported to the pharmacovigilance center of the Pharmaceuticals and Pharmacy General Directorate of the Turkish Ministry of Health. Reporting these types of side effects is important for alerting other clinicians. Patients should also be warned that the same side effects may develop with repeated doses.

\section{Ethics}

Informed Consent: Consent form was filled out by the participant.

Peer-review: Externally and internally peer-reviewed.

\section{Authorship Contributions}

Surgical and/or Medical Practices: T.N., M.I.T., O.R.S., Data Collection or Processing: T.N., T.Y., Interpretation: H.P., O.R.S., Literature Search: O.R.S., T.Y., M.I.T., Writing: T.N., M.I.T.

Conflict of Interest: No conflict of interest was declared by the authors.

Financial Disclosure: The authors declared that this study received no financial support.

\section{References}

1. Curtis BR. Drug-induced immune thrombocytopenia: incidence, clinical features, laboratory testing, and pathogenic mechanisms. Immunohematology. 2014;30:55-65.

2. Erol S. Rifampisin. Güncel Bilgiler Işıgında Antibiyotikler. Ed: Leblebicioğlu H, Usluer G, Ulusoy S. Bilimsel Tıp Kitapevi, 2008.

3. Tanrıkulu AÇ, Abakay A, Turan H. Tanrıkulu AÇ, Abakay A, Turan H. Rifampicin induced trombocyttopenia: A case report. Göztepe Tıp Dergisi. 2010;25:142-4.

4. Poole G, Stradling P, Worlledge S. Potentially serious side-effects of highdose twice-weekly rifampicin. Postgrad Med J. 1971;47:727-47.

5. Di Berardino L, Perna G, Silvestri LG. Antibodies against rifampicin in patients with tuberculosis after discontinuation of daily treatment. Am Rev Respir Dis. 1976;114:1189-90.

6. Burgess JK, Lopez JA, Gaudry LE, Chong BH. Rifampicin-dependent antibodies bind a similar or identical epitope to glycoprotein IX-specific quinine-dependent antibodies. Blood. 2000;95:1988-92.

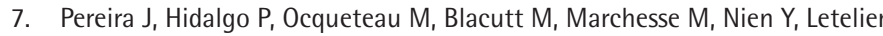
$\mathrm{L}$, Mezzano D. Glycoprotein Ib/IX complex is the target in rifampicininduced immune thrombocytopenia. Br J Haematol. 2000;110:907-10.

8. Wazny LD, Ariano RE. Evaluation and management of drug induced thrombocytopenia in the acutely ill patients. Pharmacotherapy. 2000;20:292-307.

9. Bansal R, Sharma PK, Sharma A. A case of thrombocytopenia caused by rifampicin and pyrazinamide. Indian J Pharmacol. 2013;45:405-7. 